\title{
First Higher-Multipole Model of Gravitational Waves from Spinning and Coalescing Black-Hole Binaries
}

\author{
Lionel London, ${ }^{1}$ Sebastian Khan, ${ }^{2,3}$ Edward Fauchon-Jones, ${ }^{1}$ Cecilio García, ${ }^{4}$ Mark Hannam, ${ }^{1}$ Sascha Husa, \\ Xisco Jiménez-Forteza, ${ }^{4}$ Chinmay Kalaghatgi, ${ }^{1}$ Frank Ohme, ${ }^{2,3}$ and Francesco Pannarale ${ }^{1}$ \\ ${ }^{1}$ School of Physics and Astronomy, Cardiff University, Queens Buildings, Cardiff CF24 3AA, United Kingdom \\ ${ }^{2}$ Max Planck Institute for Gravitational Physics (Albert Einstein Institute), Callinstraße 38, 30167 Hannover, Germany \\ ${ }^{3}$ Leibniz Universität Hannover, Institute for Gravitational Physics, Callinstraße 38, 30167 Hannover, Germany \\ ${ }^{4}$ Departament de Física Universiat de les Illes Balears and Institut d'Estudis Espacials de Catalunya,
} Carretera Valldemossa km 7.5, E-07122 Palma, Spain

(Received 4 August 2017; published 19 April 2018)

\begin{abstract}
Gravitational-wave observations of binary black holes currently rely on theoretical models that predict the dominant multipoles $(\ell=2,|m|=2)$ of the radiation during inspiral, merger, and ringdown. We introduce a simple method to include the subdominant multipoles to binary black hole gravitational waveforms, given a frequency-domain model for the dominant multipoles. The amplitude and phase of the original model are appropriately stretched and rescaled using post-Newtonian results (for the inspiral), perturbation theory (for the ringdown), and a smooth transition between the two. No additional tuning to numerical-relativity simulations is required. We apply a variant of this method to the nonprecessing PhenomD model. The result, PhenomHM, constitutes the first higher-multipole model of spinning and coalescing black-hole binaries, and currently includes the $(\ell,|m|)=(2,2),(3,3),(4,4),(2,1),(3,2),(4,3)$ radiative moments. Comparisons with numerical-relativity waveforms demonstrate that PhenomHM is more accurate than dominant-multipoleonly models for all binary configurations, and typically improves the measurement of binary properties.
\end{abstract}

DOI: 10.1103/PhysRevLett.120.161102

Introduction.-Gravitational waves (GWs) are our most direct means of observing black hole binary mergers [1-5]. Physical measurements from Advanced LIGO (aLIGO) and Virgo observations rely on agreement between experimental data and theoretical models of the GW signal emitted during inspiral, merger, and ringdown [3,6-8]. To date, these models include only the signals' dominant multipoles $(\ell=2,|m|=2$. This may be sufficient when the black holes (BHs) have comparable masses, or the signal is weak, but for binaries where one $\mathrm{BH}$ is more massive than the other (even by a ratio of only $1: 3[9-12]$ ), modeling the subdominant multipoles could significantly improve measurement accuracy, or avoid large biases.

Currently, higher multipoles have been modeled through merger only for nonspinning binaries $[13,14]$, or restricted corners of the parameter space [15]. Generic higher-multipole models exist only for the inspiral, e.g., Refs. [16-18]. They can also be calculated for individual binary configurations from numerical relativity (NR) simulations (see Fig. 1), but an analytic, higher-multipole model of spinning binaries would be extremely valuable. Even an approximate model would make it possible to assess the importance of higher multipoles in interpreting a GW observation, without the direct use of computationally expensive NR simulations.

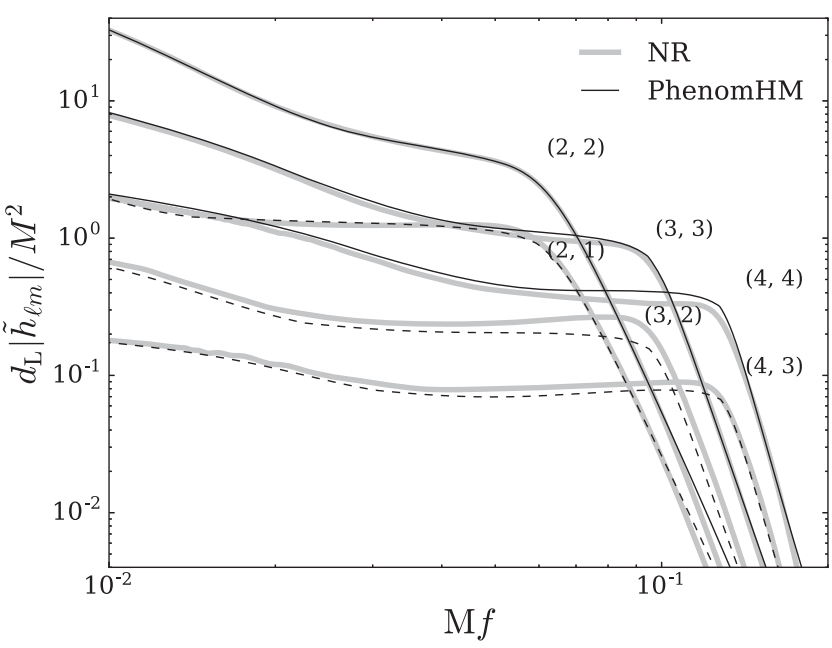

FIG. 1. A GW signal decomposed into its multipolar contributions, for a system with mass ratio $1: 8$ and spin on the larger $\mathrm{BH}$ of $\chi_{1}=\mathbf{S}_{1} / m_{1}^{2}=(0,0,-0.5)$. Our model (PhenomHM) is included as solid $(m=\ell)$ and dashed $(m=\ell-1)$ black lines. Numerical Relativity (NR)multipoles are displayed in gray, thick lines. Axes are in dimensionless units, where $M$ is the total system mass and $d_{L}$ is the source's luminosity distance. 
This need has motivated the flexible construction we present here: we use basic results from post-Newtonian (PN) and perturbation theory to map the dominant multipole into its subdominant counterparts. Our approach can be applied to any frequency-domain model, and may accelerate the further development of higher-multipole models. Here, we construct an explicit model, PhenomHM, by extending PhenomD, a non-higher-multipole model tuned to NR [19]. We demonstrate the accuracy improvement when higher multipoles are added, which, in turn, boosts our ability to recover source parameters, particularly distance and orientation. Figure 1 illustrates an application of our new model by comparing its prediction for various multipoles of the GW signal of a spinning binary with a mass ratio of $1: 8$ to the same multipoles as determined by a NR simulation. For the same system, a total mass of $90 M_{\odot}$, a distance of $500 \mathrm{Mpc}$, and an inclination of $l=\pi / 3$, Fig. 2 illustrates the impact of higher multipoles on the total GW strain, $h=h_{+}-i h_{\times}$. When compared to the dominant-multipole model, PhenomHM reproduces the signal far more accurately. This level of agreement is achieved without any additional tuning to NR waveforms.

Methods. - We consider the GW strain decomposed into spin weight -2 spherical harmonics [22]

$$
h(t, \vec{\lambda}, \theta, \phi)=\sum_{\ell \geq 2} \sum_{-\ell \leq m \leq \ell} h_{\ell m}(t, \vec{\lambda})_{-2} Y_{\ell m}(\theta, \phi),
$$
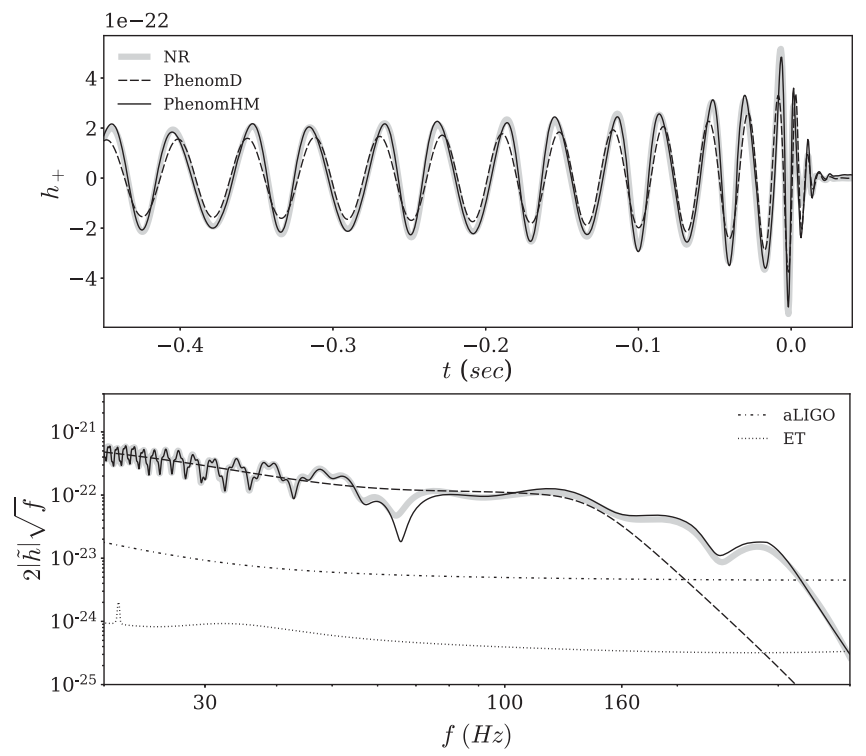

FIG. 2. The system considered in Fig. 1, with an inclination of $\imath=\pi / 3$, a total mass of $90 M_{\odot}$, and a distance of $500 \mathrm{Mpc}$. In each panel, the NR data are displayed in gray, thick lines. The PhenomHM and PhenomD models are shown in thin black lines that are continuous and dashed, respectively. Top panel: a time domain comparisons of plus polarizations. Bottom panel: a comparison of frequency domain amplitudes. Modeled aLIGO and Einstein Telescope noise spectral densities [20,21] are displayed in dashed-dotted and dotted black lines, respectively. where $t$ is the time, $\vec{\lambda}$ denotes the intrinsic parameters (masses, spins), and $\theta$ and $\phi$ are the spherical angles in a source-centered coordinate system with the its $z$ axis along the orbital angular momentum. We first describe modelagnostic transformations between the Fourier representations $\tilde{h}_{22}$ and the various subdominant multipoles $\tilde{h}_{\ell m}$ by using the analytic relationships of $\mathrm{PN}$ and $\mathrm{BH}$ perturbation theory. Figure 1 shows that all of the multipole amplitudes are qualitatively similar, suggesting that an appropriate transformation of the $(2,2)$ multipole's amplitude could conceivably be sufficient to approximate each of the other multipoles. A similar observation applies to each multipole's phase (or the phase derivative, which is often a more instructive quantity [23]). We construct a simple transformation that achieves this. We separate each $\mathrm{GW}$ multipole into amplitude $A_{\ell m}(f)$ and phase $\varphi_{\ell m}(f)$,

$$
\begin{gathered}
\tilde{h}_{\ell m}(f)=A_{\ell m}(f) \times \exp \left\{i \varphi_{\ell m}(f)\right\} \\
\approx\left|\beta_{l m}(f)\right| A_{22}\left(f_{22}^{\mathrm{A}}\right) \times \exp \left\{i\left[\kappa(f) \varphi_{22}\left(f_{22}^{\varphi}\right)+\Delta_{\ell m}(f)\right]\right\} .
\end{gathered}
$$

Equation (3) emphasizes that we construct $\tilde{h}_{\ell m}$ by mapping $f_{22}$, and the related amplitude and phase functions, $A_{22}$ and $\varphi_{22}$, into $f, A_{\ell m}(f)$, and $\varphi_{\ell m}(f)$. The frequency, amplitude, and phase transformations are simple linear mappings between the radiative mass quadrupole $\tilde{h}_{22}$ and other multipole moments [24]. For compactness, we refer to our procedure as quadrupole mapping.

Our construction is motivated by three aspects of $\mathrm{PN}$ and quasi-normal mode (QNM) theory. First, during inspiral, the time-domain oscillation frequency of each $(\ell, m)$ multipole is approximately $m \Omega$, where $\Omega$ is the binary orbital frequency. In this approximation, the frequency $f$ of each multipole corresponds to a (2,2)-multipole frequency of $2 f / \mathrm{m}$.

Second, the stationary phase approximation allows the association of these frequencies with values in $\tilde{h}_{\ell m}(f)$ 's domain [25-27]. Simultaneously, the stationary phase approximation (SPA) approximates each amplitude, beyond leading order in frequency $[17,28]$. We use the SPA amplitude $\hat{H}_{\ell m}(f)$ to appropriately rescale $\tilde{h}_{22}$ by

$$
\beta_{\ell m}(f)=\frac{\hat{H}_{\ell m}\left(f_{22}^{\mathrm{A}}\right)}{\hat{H}_{22}\left(f_{22}^{\mathrm{A}}\right)}\left(\frac{\hat{H}_{\ell m}(f)}{\hat{H}_{\ell m}(2 f / m)}\right) .
$$

With this rescaling choice we divide away the low order behavior of $\tilde{h}_{22}\left(f_{22}^{\mathrm{A}}\right)$, and then scale by $\hat{H}_{\ell m}\left(f_{22}^{\mathrm{A}}\right)$. The factor in parentheses is required to recover $\hat{H}_{\ell m}(f)$ at low frequencies. While $\hat{H}_{\ell m}(f)$ is provided in, e.g., Ref. [28] up to $2 \mathrm{PN}$ order, we use a restricted version of their results to enforce regular behavior at high frequencies. For $(\ell, m)=(2,1)$, we use $1.5 \mathrm{PN}$ order to approximate spin dependence. For the other multipoles, which depend weakly on spin effects, we use only leading PN order in $f$. Although we have presented a minimal formulation of $\beta_{\ell m}, \beta_{\ell m}=\hat{H}_{\ell m}(f) / \hat{H}_{22}\left(f_{22}^{A}\right)$ performs slightly better for spin-aligned systems. 
Lastly, QNM theory implies that ringdown frequencies of different $\tilde{h}_{\ell m}$ are related by the difference between the fundamental QNM frequencies of the $(2,2)$ and $(\ell, m)$ multipoles, $f_{\ell m}^{\mathrm{RD}}-f_{22}^{\mathrm{RD}}$.

To bridge the "gap" between the PN and QNM regimes, we find that linear interpolation is sufficient. The result of this choice is a piecewise-linear mapping,

$f_{22}(f)= \begin{cases}\frac{2}{m} f, & f \leq f_{0}, \\ \frac{f_{22}^{\mathrm{RD}}-2 f_{0} / m}{f_{\ell m}^{\mathrm{RD}}-f_{0}}\left(f-f_{0}\right)+\frac{2 f_{0}}{m}, & f_{0}<f \leq f_{\ell m}^{\mathrm{RD}}, \\ f-\left(f_{\ell m}^{\mathrm{RD}}-f_{22}^{\mathrm{RD}}\right), & f>f_{\ell m}^{\mathrm{RD}} .\end{cases}$

As is done in PhenomD, we optimized agreement with NR simulations by allowing different values of $f_{0}$ for the amplitude and phase, hence the distinction between $f_{22}^{A}$ and $f_{22}^{\varphi}$ in Eq. (3). Here, we use $f_{0}^{A}=0.018 f_{\ell m}^{\mathrm{RD}} / f_{22}^{\mathrm{RD}}$, $f_{0}^{\varphi}=0.014 f_{\ell m}^{\mathrm{RD}} / f_{22}^{\mathrm{RD}}$, and $f_{\ell m}^{\mathrm{RD}}=\omega_{\ell m 0} / 2 \pi$, where $\omega_{\ell m 0}$ is the real-valued frequency of the fundamental QNM. Equation (5) is sufficient to relate the frequency-domain phase derivatives of all multipoles to each other, $\varphi_{\ell m}^{\prime}(f) \approx \varphi_{22}^{\prime}\left[f_{22}(f)\right]$. Integrating once yields the phase relation that contains the inverse of the derivative of $f_{22}$ (where we understand the derivative at each boundary as the limit from lower frequencies toward that boundary). The additional, multipole-dependent phase offsets is determined from continuity and PN theory. The resulting coefficients read

$$
\begin{gathered}
\kappa=\frac{1}{f_{22}^{\prime}(f)}(\text { piecewise constant }), \\
\Delta_{\ell m}= \begin{cases}\frac{\pi}{2}[3 \ell+\bmod (\ell+m, 2)]-\pi, & f \leq f_{0}^{\varphi}, \\
\varphi_{\ell m}\left(f_{0}^{\varphi}\right)-\kappa \varphi_{22} 22\left[f_{22}^{\varphi}\left(f_{0}^{\varphi}\right)\right], & f_{0}^{\varphi}<f \leq f_{\ell m}^{\mathrm{RD}}, \\
\varphi_{\ell m}\left(f_{\ell m}^{\mathrm{RD}}\right)-\varphi_{22} 22\left[f_{22}^{\varphi}\left(f_{\ell m}^{\mathrm{RD}}\right)\right], & f>f_{\ell m}^{\mathrm{RD}} .\end{cases}
\end{gathered}
$$

The phase shifts introduced explicitly for $f<f_{0}^{\varphi}$ reflect mass and current multipole separation (see, e.g., Eq. (326) of Ref. [24]) as well as the necessary symmetry properties of each multipole $[24,29]$.

Equations (3)-(7) constitute a minimalistic modelagnostic method to map the dominant into subdominant multipoles.

Application to PhenomD.-Given a dominant multipole model, further refinements may be applied. We consider PhenomD [19,23]. Comparison with NR data shows that the phase resulting from Eq. (5) is least accurate for frequencies just below $f_{\ell m}^{\mathrm{RD}}$, where $f_{22}(f)$ 's linear interpolation does not ensure a simple shift from $f_{22}^{\mathrm{RD}}$ to $f_{\ell m}^{\mathrm{RD}}$, but rather a shift with some nonunity slope.

A simple extension of the PhenomD phase ansatz and a compatible adjustment of $f_{22}(f)$ for $f>f_{\ell m}^{\mathrm{RD}}$ are sufficient to impart the correct behavior prior to the ringdown frequency. In the merger-ringdown phase ansatz, Eq. (14) of Ref. [19], we add factors of $f_{22}^{\mathrm{RD}} / f_{\ell m}^{\mathrm{RD}}$ to the last term, and use the appropriate damping frequency for each QNM. The modified parts of the model are

$$
\begin{gathered}
f_{22}(f)= \begin{cases}\frac{f_{22}^{\mathrm{RD}}}{f_{\ell m}^{\mathrm{RD}}} f, & f>f_{\ell m}^{\mathrm{RD}}, \\
\text { see Eq. }(5), & \text { otherwise, }\end{cases} \\
\phi_{\mathrm{MR}}^{\ell m}(f)=\frac{1}{\eta}\left[\alpha_{0}+\alpha_{1} f-\alpha_{2} f^{-1}+\frac{4}{3} \alpha_{3} f^{3 / 4}\right. \\
\left.+\alpha_{4} \frac{f_{22}^{\mathrm{RD}}}{f_{\ell m}^{\mathrm{RD}}} \tan ^{-1}\left(\frac{f-\alpha_{5} f_{22}^{\mathrm{RD}}}{\frac{f_{22}^{\mathrm{RD}}}{f_{\ell m}^{\mathrm{RD}}} f_{\ell m}^{\mathrm{damp}}}\right)\right] .
\end{gathered}
$$

Equations (3)-(9) define PhenomHM via the mapping of PhenomD.

Results.-We compare PhenomHM to NR simulations to assess its accuracy and utility. We consider the simulations used to calibrate the dominant-multipole PhenomD model, performed with the BAM [30,31] and SPEC [32,33] codes. The simulations cover mass ratios from $1: 1$ to $1: 18$, and spin magnitudes up to 0.85 (and up to 0.98 for equal-mass configurations). We test PhenomHM in three ways. (i) We first confirm that an inverse Fourier transform of each multipole produces qualitatively correct time-domain waveforms without pathological features. (ii) We calculate a noise-weighted normalized inner product (match) between the NR waveforms and the model to estimate the accuracy of the model, which is crucial for GW search and parameter-estimation purposes. (iii) We perform parameter-estimation studies to gauge the impact of higher multipoles on GW measurements.

The match between PhenomHM and NR, $\left(h_{\mathrm{HM}} \mid h_{\mathrm{NR}}\right)$, is weighted by the anticipated aLIGO noise power spectrum at design sensitivity [20] and calculated following Eq. (2) of Ref. [34], with a starting frequency $f_{\min }=30 \mathrm{~Hz}$. The NR waveforms contain all multipoles with $\ell \leq 5$ and the PhenomHM waveforms include multipoles with $\ell=|m| \leq$ 4 and $|m|=\ell-1$. The PhenomHM template waveform is taken with the same intrinsic parameters $\left(M_{1}, M_{2}, \chi_{1}, \chi_{2}\right)$ and inclination $l$ as the NR signal and the match is optimized over the time of arrival, template polarization, and initial orbital phase.

Figure 3 presents matches for all $19 \mathrm{NR}$ waveforms used to calibrate PhenomD. The dominant-multipole-model results (left) would be almost identical for any accurate model of the $(2,2)$ multipole. As the matches vary with the source's polarization and orbital phase angles, we show average values after appropriately accounting for variations in the signal strength (see, e.g., Ref. [10]). For face-on $(l=0)$ and face-off inclinations $(l=\pi)$, the PhenomHM match (right) marginally decreases relative to the dominant-multipole model due to inaccuracies in the PhenomHM $(l, m)=$ $(3,2)$ multipole. However, PhenomHM displays consistently 

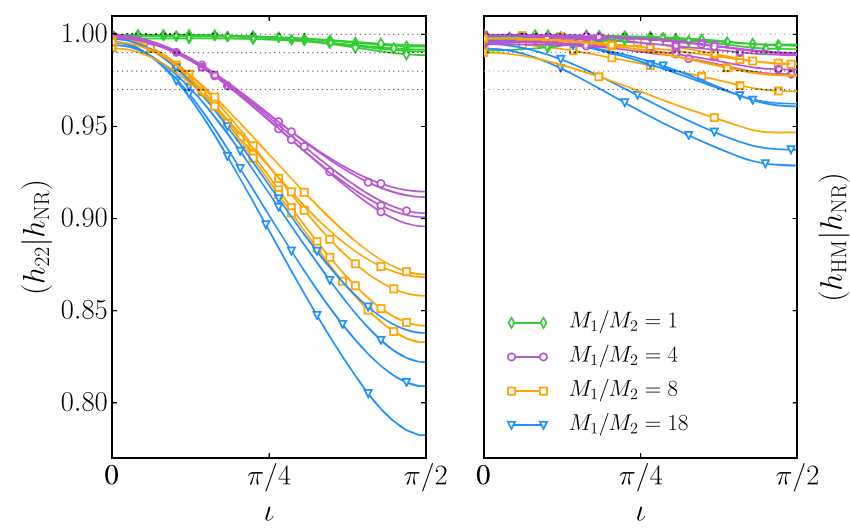

FIG. 3. Matches between models and NR. All curves are symmetric about $l=p i / 2$. The NR waveforms contain all multipoles up to $\ell=5$, while PhenomHM contains multipoles with $\ell=|m| \leq 4$ and $|m|=\ell-1$. Each curve corresponds to a NR simulation within the PhenomD calibration region [19] scaled to $100 M_{\odot}$ with a minimum frequency of $30 \mathrm{~Hz}$. Higher multipoles are not significant for configurations with $M_{1} / M_{2}=$ 1 (green curves with diamond markers), as opposed to cases with $M_{1} / M_{2}=4$ (purple curves with circles), and especially $M_{1} / M_{2}=8$ (orange curves with squares) and $M_{1} / M_{2}=18$ (blue curves with triangles). Each mass-ratio set contains systems with varying nonprecessing spins [19]. Left panel: average matches between NR and a model with only $l=|m|=2$ multipoles. While we used PhenomD, these results are common to all models that lack higher multipoles. Right panel: matches between NR and PhenomHM, which shows significant improvement.

higher matches than a dominant-multipole model for all inclined systems. As the mass ratio increases, the performance of the dominant-multipole model rapidly degrades for edge-on configurations, but remains high for PhenomHM.

For nonspinning systems, PhenomHM typically has matches higher than 0.99 for mass ratios less than or equal to 8 . The matches degrade for high-mass-ratio, high-alignedspin systems with edge-on inclination, and the match average

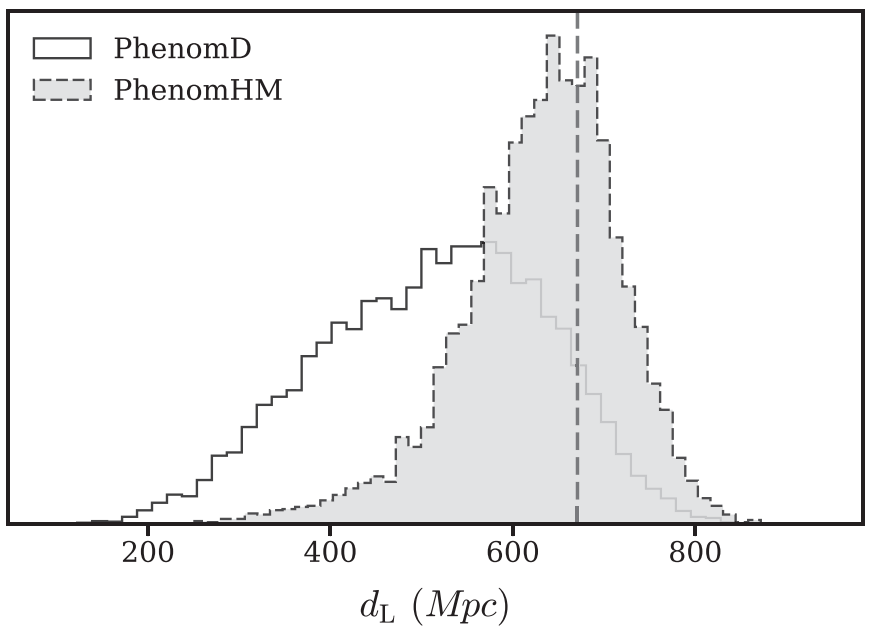

over polarization and source orbital phase can be as low as 0.93 , for a mass-ratio $1: 18$ system with $\chi_{1}=0.4$. However, the worst matches correspond to inclinations that suppress the dominant mode, making these signals significantly weaker, and therefore less likely to be observed.

As a more detailed check, we calculated multipole-bymultipole matches between NR and PhenomHM for each waveform. Most individual multipoles match at 0.99 or better. The quality of agreement degrades for high aligned spin. Discounting cases with component spins of 0.75 or greater, the average match is $\sim 0.98$ for $(\ell, m)=(4,4)$, and 0.99 and above for all other $\ell=m$ cases. Sphericalspheroidal mixing significantly impacts $(3,2)$ and $(4,3)$, so their average match is $\sim 0.92$ [35]. We also broadly checked the accuracy of the individual multipole amplitudes by comparing the signal-to-noise ratio (SNR) in each between the NR and PhenomHM results. The subdominant multipoles typically have amplitude errors much less than $15 \%$, which we consider acceptable, given that our goal was to achieve an order-of-magnitude estimate.

We expect that the main value of PhenomHM will be in parameter recovery. To assess this, we injected NR waveforms in zero noise [36] and performed a parameter recovery analysis similar to Ref. [37] with PhenomD and PhenomHM using LALINFERENCE $[6,38,39]$. Our injected signals have a network SNR of $\sim 25$. For configurations with a variety of mass ratios and spins, the inaccuracies in PhenomHM did not lead to appreciable biases in recovering masses and spins for SNRs of $\sim 25$. A more detailed parameter-estimation study is in preparation.

Relative to PhenomD, PhenomHM can significantly improve source inclination measurements. This is not surprising: higher multipoles have an inclination dependent impact on signal morphology, as seen in Fig. 2 for a specific case. In Fig. 4 we show an example of a $100 M_{\odot}$ binary with mass ratio $1: 4$ and spins $\chi_{1}=\chi_{2}=0.5$, at a distance of

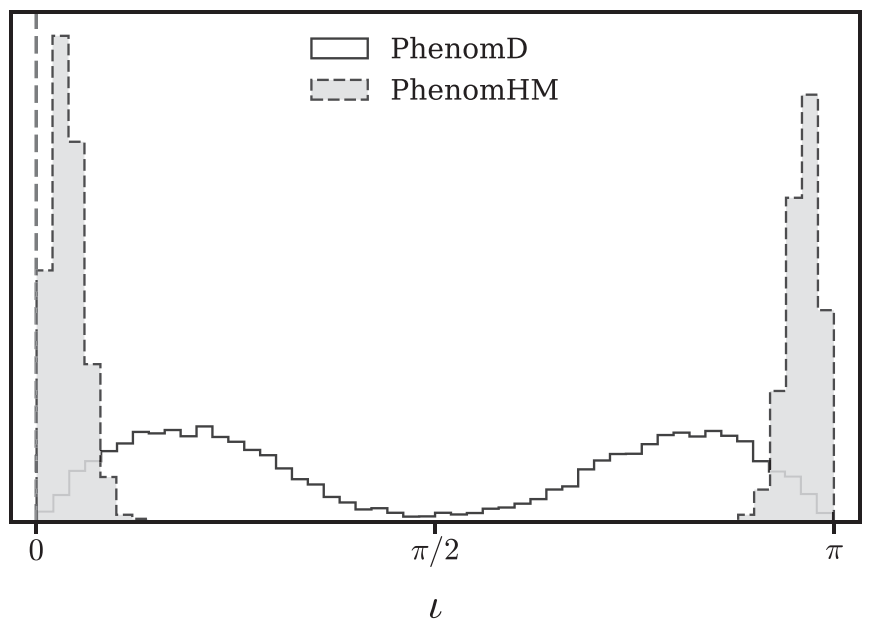

FIG. 4. Parameter recovery for a $100 M_{\odot}$ mass-ratio $1: 4$ binary, with aligned spins $\chi_{1}=\chi_{2}=0.5$, optimally oriented to the detector at a distance of $671 \mathrm{Mpc}$. The higher-multipole PhenomHM model allows us to correctly identify the source orientation and to reduce the uncertainty in distance by approximately $40 \%$. 
$671 \mathrm{Mpc}$. Since systems with inclination angles near 0 or $\pi$ are roughly twice as strong as edge-on systems, they can be observed in a volume of the Universe 8 times larger; thus we inject the signal face on to the detector. Using PhenomD, we recover only our prior expectation for the inclination, and the $90 \%$ credible region for the distance ranges from 299 to $702 \mathrm{Mpc}$. All GW observations to date display results similar to this [2-6]. However, with PhenomHM the binary inclination angle is recovered with an uncertainty of only $0.21 \mathrm{rad}(12 \mathrm{deg})$. The uncertainty in distance is reduced by $30 \%$, with the $90 \%$ credible region ranging from 475 to $757 \mathrm{Mpc}$. Here, distance and inclination uncertainty are dominated by uncertain sky localization, which is drastically reduced with a three-detector network $[5,40,41]$.

Discussion.-We presented a simple and flexible method to transform the dominant GW multipole into higher multipoles for nonprecessing binary BH systems. This may be applied to any dominant-multipole-only frequency-domain model. We introduced the first application of this method to the phenomenological model PhenomD [19,23], and produced a more accurate higher-multipole model, which we call PhenomHM.

Across the entire calibration region of the underlying PhenomD, mass ratios up to $1: 18$, and spins up to 0.85 , PhenomHM agrees better with NR waveforms than the dominant-multipole-only models. In a first set of parameterestimation tests, even for face-on systems, where the highermultipole contribution to the signal is weak, PhenomHM yields a dramatic improvement over PhenomD in recovering the source inclination and distance.

It is striking that simple approximations can be used to model the subdominant multipoles. In particular, simple linear transformations are sufficient to capture the qualitative behavior of the signal throughout inspiral, (nonlinear) merger, and ringdown. This approach is a means to rapidly extend any dominant-multipole model to higher multipoles (including models that treat precession). An extension of PhenomHM to precession will be presented in the near future.

Despite its encouraging performance, further studies are needed to fully quantify the value of PhenomHM in GW astronomy. The most obvious next step is to use PhenomHM as the basis for a precise tuning of the subdominant multipoles to NR waveforms. This work is underway. Several physical features are also absent from PhenomHM. The most notable is the mixing between $|m|=\ell$ and $|m|=\ell-1$ multipoles through merger and ringdown [35,42]. An obvious extension to precessing systems, following the prescription of PhenomP [43], would also neglect to model the asymmetry between $m>0$ and $m<0$ multipoles that leads to out-of-plane recoil [44].

However, given that the model captures the phenomenology of the subdominant multipoles across the binary $\mathrm{BH}$ parameter space, and shows mismatch errors of at most a few percent, and for much of the parameter space less than $1 \%$, PhenomHM will make it possible to assess the importance of subdominant multipoles in GW observations, and improve the accuracy of parameter estimates. For highmass binaries, where the merger and ringdown dominate the signal, it will also be valuable in strengthening current tests of general relativity.

We thank Geraint Pratten for useful discussions. We thank P. Ajith and Chandra Mishra for useful discussions and for sharing a draft on their recently completed nonspinning higher multipole model. We also thank R. Cotesta, A. Bohé, A. Buonanno, and A. Taracchini for making us aware of their progress towards a higher multipole model in the EOBNR framework. The work presented in this Letter was supported by Science and Technology Facilities Council (STFC) Grant No. ST/L000962/1, European Research Council Consolidator Grant No. 647839, the Max Planck Society, the Max Planck Prince of Asturias Mobility Award, Spanish Ministry of Economy and Competitiveness Grants No. CSD2009-00064, No. FPA2013-41042-P, and No. FPA2016-76821-P, the Spanish Agencia Estatal de Investigación, European Union FEDER funds, Vicepresidència i Conselleria d'Innovació, Recerca i Turisme, Conselleria d'Educació, i Universitats del Govern de les Illes Balears, and the Fons Social Europeu. BAM simulations were carried out at Advanced Research Computing (ARCCA) at Cardiff, as part of the European PRACE petascale computing initiative on the clusters Hermit, Curie and SuperMUC, on the UK DiRAC Datacentric cluster, and on the BSC MareNostrum computer under PRACE and RES (Red Española de Supercomputación) allocations.

[1] B. P. Abbott et al., Observation of Gravitational Waves from a Binary Black Hole Merger, Phys. Rev. Lett. 116, 061102 (2016).

[2] B. P. Abbott et al., GW151226: Observation of Gravitational Waves from a 22-Solar-Mass Binary Black Hole Coalescence, Phys. Rev. Lett. 116, 241103 (2016).

[3] B. P. Abbott et al., GW170104: Observation of a 50-SolarMass Binary Black Hole Coalescence at Redshift 0.2, Phys. Rev. Lett. 118, 221101 (2017).

[4] B. P. Abbott et al., GW170814: A Three-Detector Observation of Gravitational Waves from a Binary Black Hole Coalescence, Phys. Rev. Lett. 119, 141101 (2017).

[5] B. P. Abbott et al., GW170608: Observation of a 19-solarmass binary black hole coalescence, 2017.

[6] B. P. Abbott et al., Properties of the Binary Black Hole Merger GW150914, Phys. Rev. Lett. 116, 241102 (2016).

[7] T. D. Abbott et al., Improved Analysis of GW150914 Using a Fully Spin-Precessing Waveform Model, Phys. Rev. X 6, 041014 (2016).

[8] B. P. Abbott et al., Binary Black Hole Mergers in the First Advanced LIGO Observing Run, Phys. Rev. X 6, 041015 (2016).

[9] C. Capano, Y. Pan, and A. Buonanno, Impact of higher harmonics in searching for gravitational waves from 
nonspinning binary black holes, Phys. Rev. D 89, 102003 (2014).

[10] V. Varma and P. Ajith, Effects of nonquadrupole modes in the detection and parameter estimation of black hole binaries with nonprecessing spins, 2016.

[11] J. C. Bustillo, P. Laguna, and D. Shoemaker, Detectability of gravitational waves from binary black holes: Impact of precession and higher modes, Phys. Rev. D 95, 104038 (2017).

[12] J. Lange et al., A parameter estimation method that directly compares gravitational wave observations to numerical relativity, 2017.

[13] Y. Pan, A. Buonanno, M. Boyle, L. T. Buchman, L. E. Kidder, H. P. Pfeiffer, and M. A. Scheel, Inspiral-mergerringdown multipolar waveforms of nonspinning black-hole binaries using the effective-one-body formalism, Phys. Rev. D 84, 124052 (2011).

[14] A. K. Mehta, C. K. Mishra, V. Varma, and P. Ajith, Accurate inspiral-merger-ringdown gravitational waveforms for nonspinning black-hole binaries including the effect of subdominant modes, Phys. Rev. D 96, 124010 (2017).

[15] J. Blackman, S. E. Field, M. A. Scheel, C. R. Galley, C. D. Ott, M. Boyle, L. E. Kidder, H. P. Pfeiffer, and B. Szilágyi, A numerical relativity waveform surrogate model for generically precessing binary black hole mergers, 2017.

[16] K. G. Arun, A. Buonanno, G. Faye, and E. Ochsner, Higherorder spin effects in the amplitude and phase of gravitational waveforms emitted by inspiraling compact binaries: Readyto-use gravitational waveforms, Phys. Rev. D 79, 104023 (2009); Erratum, 84, 049901 (2011).

[17] K. Chatziioannou, A. Klein, N. Cornish, and N. Yunes, Analytic Gravitational Waveforms for Generic Precessing Binary Inspirals, Phys. Rev. Lett. 118, 051101 (2017).

[18] A. Klein, N. Cornish, and N. Yunes, Fast frequency-domain waveforms for spin-precessing binary inspirals, Phys. Rev. D 90, 124029 (2014).

[19] S. Khan, S. Husa, M. Hannam, F. Ohme, M. Pürrer, X. Jiménez Forteza, and A. Bohé, Frequency-domain gravitational waves from nonprecessing black-hole binaries. II. A phenomenological model for the advanced detector era, Phys. Rev. D 93, 044007 (2016).

[20] D. Shoemaker et al., Advanced ligo anticipated sensitivity curves, LIGO-T0900288, https://dcc.ligo.org/cgi-bin/ DocDB/ShowDocument?docid=2974, 2010.

[21] S. Hild et al., Sensitivity studies for third-generation gravitational wave observatories, Classical Quantum Gravity 28, 094013 (2011).

[22] J. N. Goldberg, A. J. MacFarlane, E. T. Newman, F. Rohrlich, and E. C. G. Sudarshan, Spin s spherical harmonics and edth, J. Math. Phys. (N.Y.) 8, 2155 (1967).

[23] S. Husa, S. Khan, M. Hannam, M. Pürrer, F. Ohme, X. Jiménez Forteza, and A. Bohé, Frequency-domain gravitational waves from nonprecessing black-hole binaries. I. New numerical waveforms and anatomy of the signal, Phys. Rev. D 93, 044006 (2016).

[24] L. Blanchet, Gravitational radiation from post-newtonian sources and inspiralling compact binaries, Living Rev. Relativity 17, 2 (2014).
[25] B. S. Sathyaprakash and S. V. Dhurandhar, Choice of filters for the detection of gravitational waves from coalescing binaries, Phys. Rev. D 44, 3819 (1991).

[26] L. S. Finn and D. F. Chernoff, Observing binary inspiral in gravitational radiation: One interferometer, Phys. Rev. D 47, 2198 (1993).

[27] T. Damour, B. R. Iyer, and B. S. Sathyaprakash, Frequency domain $\mathrm{P}$ approximant filters for time truncated inspiral gravitational wave signals from compact binaries, Phys. Rev. D 62, 084036 (2000).

[28] C. K. Mishra, A. Kela, K. G. Arun, and G. Faye, Ready-touse post-Newtonian gravitational waveforms for binary black holes with nonprecessing spins: An update, Phys. Rev. D 93, 084054 (2016).

[29] L. E. Kidder, Using full information when computing modes of post-Newtonian waveforms from inspiralling compact binaries in circular orbit, Phys. Rev. D 77, 044016 (2008).

[30] B. Brügmann, J. A. González, M. Hannam, S. Husa, U. Sperhake, and W. Tichy, Calibration of moving puncture simulations, Phys. Rev. D 77, 024027 (2008).

[31] S. Husa, J. A. González, M. Hannam, B. Brügmann, and U. Sperhake, Reducing phase error in long numerical binary black hole evolutions with sixth order finite differencing, Classical Quantum Gravity 25, 105006 (2008).

[32] M. A. Scheel, H. P. Pfeiffer, L. Lindblom, L. E. Kidder, O. Rinne, and S. A. Teukolsky, Solving Einstein's equations with dual coordinate frames, Phys. Rev. D 74, 104006 (2006).

[33] http://www.black-holes.org/waveforms.

[34] I. Harry, S. Privitera, A. Bohé, and A. Buonanno, Searching for gravitational waves from compact binaries with precessing spins, Phys. Rev. D 94, 024012 (2016).

[35] L. London, D. Shoemaker, and J. Healy, Modeling ringdown: Beyond the fundamental quasinormal modes, Phys. Rev. D 90, 124032 (2014); Erratum, 94, 069902 (2016).

[36] P. Schmidt, I. W. Harry, and H. P. Pfeiffer, Numerical relativity injection infrastructure, 2017.

[37] B. P. Abbott et al., Effects of waveform model systematics on the interpretation of GW150914, Classical Quantum Gravity 34, 104002 (2017).

[38] J. Veitch et al., Parameter estimation for compact binaries with ground-based gravitational-wave observations using the LALINFERENCE software library, Phys. Rev. D 91, 042003 (2015).

[39] https://wiki.ligo.org/DASWG/LALSuite.

[40] B. P. Abbott et al., Prospects for observing and localizing gravitational-wave transients with Advanced LIGO and Advanced Virgo, Living Rev. Relativity 19, 1 (2016).

[41] B. Abbott et al., GW170817: Observation of Gravitational Waves from a Binary Neutron Star Inspiral, Phys. Rev. Lett. 119, 161101 (2017).

[42] L. T. London, Modeling ringdown II: nonprecessing binary black holes, 2018.

[43] M. Hannam, P. Schmidt, A. Bohé, L. Haegel, S. Husa, F. Ohme, G. Pratten, and M. Pürrer, Simple Model of Complete Precessing Black-Hole-Binary Gravitational Waveforms, Phys. Rev. Lett. 113, 151101 (2014).

[44] B. Brügmann, J. A. González, M. Hannam, S. Husa, and U. Sperhake, Exploring black hole superkicks, Phys. Rev. D 77, 124047 (2008). 